\title{
Synthesis of quinolinequinone derivatives and related carbocyclic compounds
}

\author{
Samuel Attah $\mathrm{Egu}^{1}$, Uchechukwu Chris Okoro ${ }^{1}$, and Thomas Wirth ${ }^{2 *}$ \\ ${ }^{1}$ Department of Pure and Industrial Chemistry, University of Nigeria, Nsukka, Nigeria \\ ${ }^{2}$ School of Chemistry, Cardiff University, Park Place, Main Building, Cardiff CF10 3AT, UK \\ *Corresponding author's e-mail address: wirth@cf.ac.uk
}

Published online: 19 December 2014 (version 1)

Cite as: Egu et al., ScienceOpen Research 2015 (DOI: 10.14293/S2199-1006.1.SOR-CHEM.AALL9P.v1)

Reviewing status: Please note that this article is under continuous review. For the current reviewing status and the latest referee's comments please click here or scan the QR code at the end of this article.

Primary discipline: Chemistry

Keywords: Pd catalysis, amination, Buchwald-Hartwig reaction, quinolinequinones

\begin{abstract}
Palladium-catalyzed Buchwald-Hartwig aminations of various quinolinequinone derivatives give excellent yields of novel 6-arylamino derivatives of the disubstituted quinolinequinones and 3-arylamino derivatives of the corresponding naphthoquinones. The precursor quinolinequinones are prepared in a three-step sequence from 8-hydroxyquinoline. The transitionmetal-catalyzed arylations of 6,7-dibromo-5,8-quinolinequinone, 6,7-dichloro-5,8-quinolinequinone and 2,3-dichloro-1,4naphthoquinone are reported for the first time and offer fast and easy access to their derivatives.
\end{abstract}

\section{INTRODUCTION}

Quinolinequinones $\mathbf{1}$ are bicyclic heterocycles and quinone derivatives consisting of a pyridine ring and a quinone. For a long time, they have been in the focus of a large number of studies because of their wide spectrum of biological activities, which include potent antifungal, antibacterial [1], antimalarial [2] and antineoplastic [3] properties.

They are also an important structural moiety in a number of more complex antibiotic agents such as streptonigrin 2 [4], streptonigrone 3 [5] and lavendamycin 4 [6] as they play an important role in determining their biological activities [7]. The general structure of quinolinequinones $\mathbf{1}$ has been modified at various positions, and the synthesis and biological activities of various substituted quinolinequinones have been reported, often prepared by the regioselective nucleophilic substitution of 6,7-dichloro-5,8-quinolinequinone with arylamines (Figure 1).

In 1994, Ryu and Kim prepared and investigated 6-( $N$-arylamino)-7-chloro-5,8-quinolinedione derivatives and found that these compounds exhibit potent antifungal and antibacterial activities [8]. In another development, the synthesis and biological activities of several disubstituted quinoline-5,8-diones were reported [9]. The majority of these compounds were prepared by regioselective nucleophilic substitution of quinolinequinone and naphthoquinone with arylamines.

Herein we report on the synthesis of some novel derivatives of 6,7-dibromo-5,8-quinolinequinone, using a transition-metalbased approach, in order to reveal easier routes by which a number of novel derivatives of quinolinequinones can be prepared.

The Buchwald-Hartwig amination is a palladium-catalyzed cross-coupling reaction of an aryl halide or pseudo-halide with an amine together with a strong base [10]. The formation of $\mathrm{C}-\mathrm{N}$ bonds is the major focus of this research, coupling aryl halides as key intermediates with anilines. We have used various anilines in this research to demonstrate easier routes for the preparation of new quinolinequinones using a transition-metal-based approach. The Buchwald-Hartwig reaction is initiated by an oxidative addition of the aryl halide to the palladium, which is followed by coordination of the amine. The strong base then abstracts a proton from the amine, which attacks the palladium with the halide acting as a leaving group. Reductive elimination then produces the final aryl amine product and regenerates the catalyst $[11,12]$.

\section{RESULTS AND DISCUSSION}

The yields in the synthesis of target molecules using the Buchwald-Hartwig reaction depend largely on the reaction conditions and on the type of ligands used. Several ligands had been employed in C-N cross-coupling processes. Also several palladium sources have been used. We started our investigations using the combination of palladium(II) acetate and triphenylphosphine, but these conditions only provided traces of the desired coupling products. Following the discovery of Huang et al. [13] that biaryl dialkylphosphines are excellent ligands in $\mathrm{C}-\mathrm{N}$ bond formation, we exchanged triphenylphosphine with the advanced ligands Xphos and Brettphos in the reaction while still maintaining palladium(II) acetate as the palladium source. These gave very encouraging outcomes as 
<smiles>COC1=C(N)C(=O)c2nc(-c3nc(C(=O)O)c(C)c(-c4ccc(OC)c(OC)c4O)c3N)ccc2C(=O)C1=O</smiles>

1
Streptonigrin 2<smiles>COC1=C(N)C(=O)c2nc(-c3[nH]c(=O)c(C)c(-c4ccc(OC)c(OC)c4O)c3N)ccc2C1=O</smiles>

Streptonigrone 3<smiles>Cc1c(C(=O)O)nc(-c2ccc3c(n2)C(=O)C(N)=CC3=O)c2[nH]c3ccccc3c12</smiles>

Lavendamycin 4

Figure 1. Quinolinequinone derivatives.

the yields and reaction times were greatly improved (Table 1 , entry 2). This finding agrees with the discovery made by Galardon et al. [14] that the use of bulky ligands accelerates the elimination step in palladium-catalyzed reactions. We then realized the work of Brett et al. [15], describing a procedure that maximizes the efficiency of biaryl dialkylphosphine ligands. They discovered that a highly active catalyst can be generated by heating palladium(II) acetate (1 mol\%), water (4 mol\%) and Xphos (3 mol\%) for $1 \mathrm{~min}$ at $80{ }^{\circ} \mathrm{C}$ in 1,4-dioxane, which can be monitored visually by a colour change. We adopted the procedure with little variation and received the most encouraging outcome. Under these reaction conditions, the reactions were completed much faster and with similar efficiency (Table 1, entry 3; Scheme 1).<smiles>[R]C1=C([R])C(=O)c2ncccc2C1=O</smiles>

Scheme 1. Amination of 6,7-dichloro-5,8-quinolinequinone 5a.

The reaction on the quinolinequinone $\mathbf{5}$ is typically at the 6position through the involvement of the electron-donating nitrogen atom leading to compounds of type 6 [16]. In Table 2 the optimized reaction conditions have been employed for the rapid synthesis of several novel quinolinequinone

Table 1. Optimization of reaction conditions for the amination of disubstituted-5,8-quinolinequinone.

\begin{tabular}{llcc}
\hline Entry & \multicolumn{1}{c}{ Reaction conditions } & Yield (\%) & Reaction time (h) \\
\hline 1 & $\mathrm{Pd}(\mathrm{OAc})_{2} / \mathrm{PPh}_{3}$ & Trace & $28-48$ \\
2 & $\mathrm{Pd}(\mathrm{OAc})_{2} / \mathrm{BrettPhos}$ or $\mathrm{XPhos}_{3}$ & $>80$ & $12-24$ \\
3 & $\mathrm{Pd}(\mathrm{OAc})_{2} / \mathrm{XPhos}$, water activation & $>80$ & $1-3$ \\
\hline
\end{tabular}

derivatives. Both 6,7-dichloro- and 6,7-dibromo-5,8-quinolinequinones $\mathbf{5 a}$ and $\mathbf{5 b}$ have been employed in the reaction, the dibromo derivative showing slightly higher reactivity in the reaction.

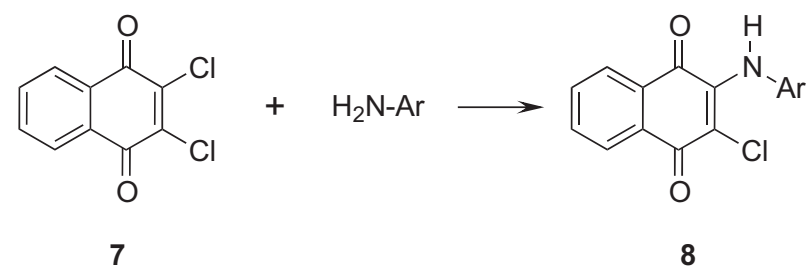

The same reaction conditions were subsequently used to functionalize 2,3-dichloro-1,4-naphthoquinone with similar efficiency as shown in Table 3.

In summary, we have developed an efficient protocol for the synthesis of different novel monosubstitued and disubstituted quinolinequinones and naphthoquinones as potential core structures for the development of biologically active molecules.

\section{EXPERIMENTAL}

\section{General}

Melting points were determined with a Fisher-John apparatus and are uncorrected. Ultraviolet and visible spectra were recorded on a Unico-UV2102 PC spectrophotometer using matched 1-cm quartz cells using methanol and DMSO as solvents. The absorption maxima are given in nanometers and the figures in parenthesis are dimethyl sulphoxide values. The nuclear magnetic resonance (NMR) spectra were recorded on Bruker DPX-250, Bruker DPX-400, Bruker DPX-500 or Oxford 300. Chemical shifts are reported in ppm values relative to tetramethylsilane (TMS) as an internal standard. Elemental analyses were obtained on Heraeus $\mathrm{CHN}-\mathrm{O}$ rapid analyser.

\section{6,7-Dichloro-5,8-quinolinequinone 5a}

The synthesis of this compound followed the same procedure as that described for $\mathbf{5 b}$; in the last step, $\mathrm{HBr}$ and $\mathrm{KBrO}_{3}$ were replaced with $\mathrm{HCl}$ and $\mathrm{KClO}_{3}$ (m.p. 243-245 ${ }^{\circ} \mathrm{C}$ ). 
Table 2. Amination of 6,7-disubstituted-5,8-quinolinequinones with different aniline derivatives.

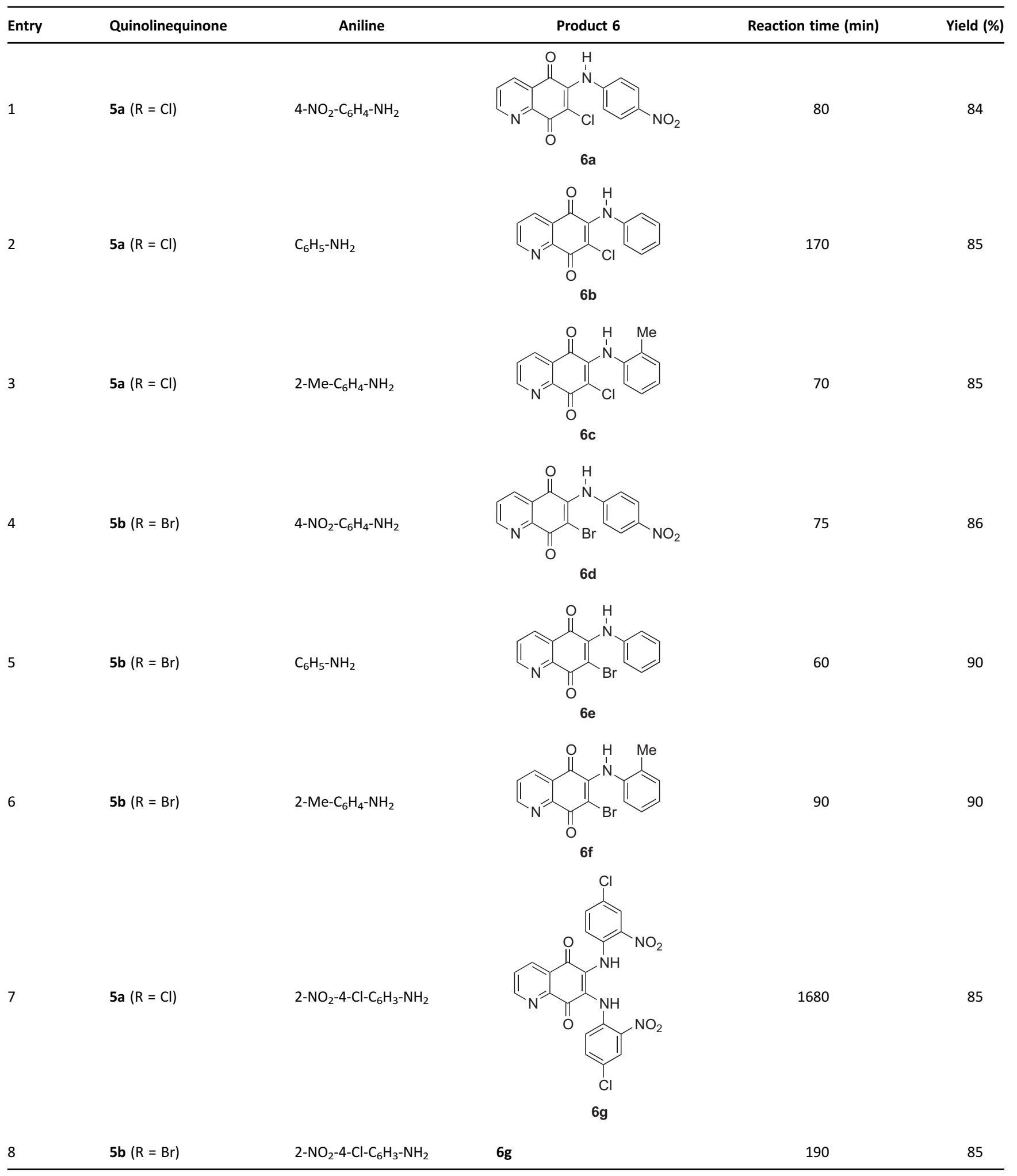


Table 3. Amination of 2,3-dichloro-1,4-naphthoquinone with different aniline derivatives.

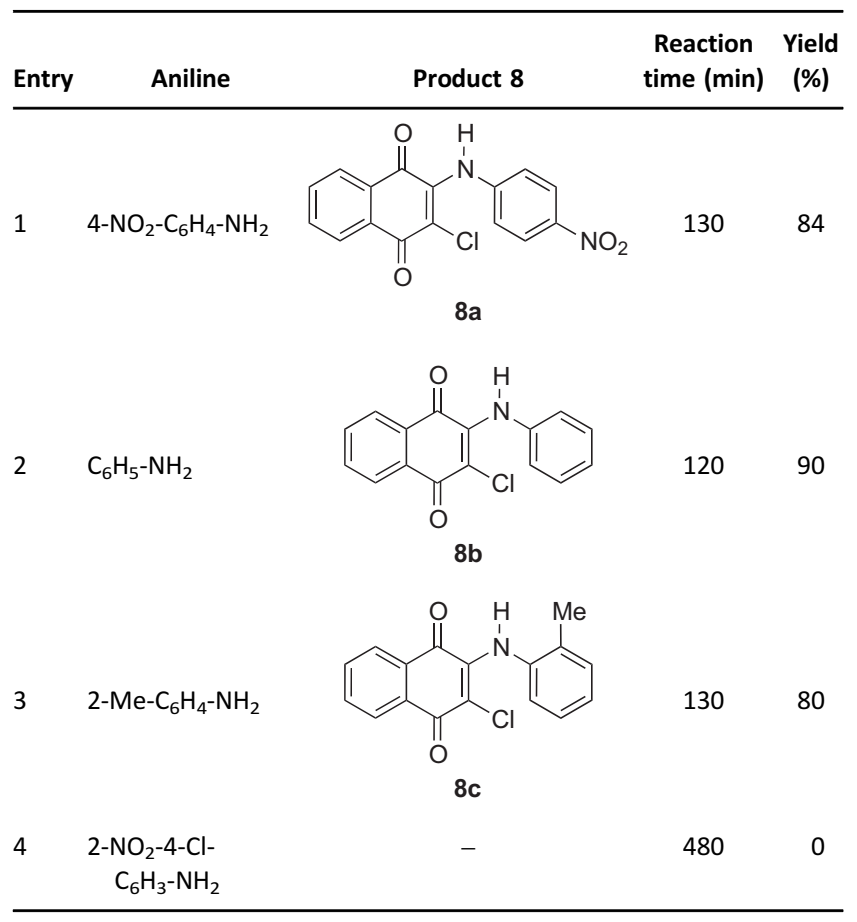

\section{6,7-Dibromo-5,8-quinolinequinone $5 b$}<smiles>Oc1cccc2cccnc12</smiles>
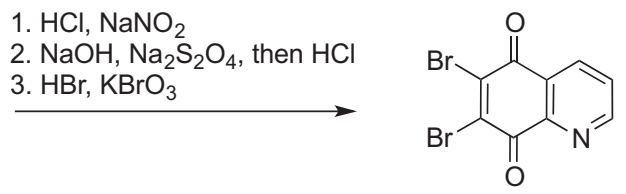

$5 b$

The synthesis of this compound was carried out in three steps reactions from 8-hydroxyquinoline with concentrated hydrochloric acid followed by the addition of sodium nitrite at $0{ }^{\circ} \mathrm{C}$ for $1 \mathrm{~h}$. The mixture was allowed to stand overnight at $0{ }^{\circ} \mathrm{C}$, washed and dried to give 8-hydroxy-5-nitrosoquinoline hydrochloride, a bright yellow solid (m.p. 179-181; lit. $\left.180{ }^{\circ} \mathrm{C}\right)[9]$.

8-Hydroxy-5-nitrosoquinoline hydrochloride $(0.216 \mathrm{~mol}, 40$ g) was added to a mixture of water $(160 \mathrm{~mL})$ and $5 \mathrm{~N} \mathrm{NaOH}$ $(260 \mathrm{~mL})$ and heated to $40{ }^{\circ} \mathrm{C} . \mathrm{Na}_{2} \mathrm{~S}_{2} \mathrm{O}_{4}(1.28 \mathrm{~mol}, 95 \mathrm{~g})$ was added to the reaction mixture and then the temperature was increased to $75{ }^{\circ} \mathrm{C}$. The reaction mixture was cooled to $50{ }^{\circ} \mathrm{C}$ and $12 \mathrm{~N} \mathrm{HCl}(250 \mathrm{~mL})$ was added. Then the reaction mixture was cooled to $0{ }^{\circ} \mathrm{C}$ and filtered to give 5 -amino-8-hydroxyquinoline dihydrochloride (34 g, 91\%) [9].

Addition of 5-amino-8-hydroxyquinoline dihydrochloride (55.6 mmol, $9 \mathrm{~g}$ ) to hydrobromic acid $(81 \mathrm{~g}$ ); the reaction mixture was heated to $60{ }^{\circ} \mathrm{C}$ and $\mathrm{KBrO}_{3}(27 \mathrm{mmol}, 4.5 \mathrm{~g})$ was added. The reaction mixture was stirred for $30 \mathrm{~min}$ at $50{ }^{\circ} \mathrm{C}$, filtered and recrystallized twice from butanol to yield 6,7dibromo-5,8-quinolinequinone as a yellow precipitate $(15.8 \mathrm{~g}$, 90\%) (m.p. 239-241; lit. 243-245 º) [9].

\section{General procedures for the Buchwald-Hartwig amination}

Procedure A: BrettPhos (0.94 $\mu \mathrm{mol}, 0.5 \mathrm{mg}, 0.15 \mathrm{~mol} \%)$ and $\mathrm{Pd}(\mathrm{OAc})_{2}(0.05 \mathrm{~mol} \%, 0.1 \mathrm{mg}, 0.05 \mathrm{~mol} \%)$ were placed in a $25 \mathrm{~mL}$ three-neck round-bottom flask. After purging with nitrogen for $30 \mathrm{~s}, 1 \mathrm{~mL}$ water and $5 \mathrm{~mL}$ EtOH were added and the solution was heated for $60 \mathrm{~s}$ to $80^{\circ} \mathrm{C}$; the preactivation could be followed by a colour change. Then 6,7-dihalo5,8-quinolinequinone, aniline derivative, base and $5 \mathrm{~mL} \mathrm{EtOH}$ were added. The reaction mixture was heated at reflux with vigorous stirring for the indicated time. The completion of the reaction was monitored by thin-layer chromatography (TLC), then cooled, filtered and recrystallized with water and acetone.

Procedure B: XPhos (0.0032 mmol, $1.5 \mathrm{mg}, 1 \mathrm{~mol} \%)$ and $\mathrm{Pd}(\mathrm{OAc})_{2}(0.0095 \mathrm{mmol}, 2.1 \mathrm{mg}, 3 \mathrm{~mol} \%)$ were placed in a $25 \mathrm{~mL}$ three-neck round-bottom flask. After purging with nitrogen for $30 \mathrm{~s}, 1 \mathrm{~mL}$ water and $5 \mathrm{~mL}$ EtOH were added and the solution was heated for $60 \mathrm{~s}$ to $80^{\circ} \mathrm{C}$; the preactivation could be followed by a colour change. Then the 6,7dihalo-5,8-quinolinequinone, aniline derivative, base and $2 \mathrm{ml}$ dioxane were added. The reaction mixture was heated at reflux with vigorous stirring for the indicated time. The completion of the reaction was monitored by TLC, then cooled, filtered and recrystallized with water and acetone.

7-Chloro-6-[(4-nitrophenyl)amino]quinoline-5,8-dione 6a Procedure A. 6,7-Dichloro-5,8-quinolinequinone 5a $(0.88$ mmol, $200 \mathrm{mg}$ ), 4-nitroaniline ( $3.52 \mathrm{mmol}, 486 \mathrm{mg}$ ), NaOt-Bu (1.06 mmol, $101 \mathrm{mg}, 1.2 \mathrm{eq}$ ), $80 \mathrm{~min}$. The product (red solid) m.p. $318-320{ }^{\circ} \mathrm{C}$ was obtained in $244 \mathrm{mg}(0.74 \mathrm{mmol})$; yield: $84 \%$.

The ultraviolet maximum absorption bands in methanol: $\mathrm{nm}$ (logE), 600 (0127), 620 (0.088), 630 (0.071), $640(0.057) ;{ }^{1} \mathrm{H}$ NMR (400 MHz, $\left.\mathrm{CDCl}_{3}\right): \delta=9.1(\mathrm{~d}, J=3.6 \mathrm{~Hz}, 1 \mathrm{H}), 8.4(\mathrm{dd}, J=$ $1.6, J=8 \mathrm{~Hz}, 1 \mathrm{H}), 8.2(\mathrm{dd}, 2 \mathrm{H}), 7.6(\mathrm{dd}, J=4.8, J=8.4 \mathrm{~Hz}, 2 \mathrm{H})$, $7.1(\mathrm{~m}, 2 \mathrm{H}) \mathrm{ppm} ;{ }^{13} \mathrm{C}$ NMR $\left(100 \mathrm{MHz}, \mathrm{CDCl}_{3}\right): \delta=183.44(\mathrm{C}=$ $\mathrm{O}(\mathrm{C}-5)), 172.38(\mathrm{C}=\mathrm{O}(\mathrm{C}-8)), 160.87(\mathrm{C}-1), 153.90$ (C-7), 151.34 (C-10), 147.15 (C-3), 133.82 (C-13), 133.65 (C-4 \& C2), 132.13 (C-12 \& C-14), 106.508 (C-11 \& C-15) ppm; IR 1670 ( $\mathrm{C}=0), 1496\left(\mathrm{NO}_{2}\right) \mathrm{cm}^{-1}$; MS 283.27, 328.02; Anal. Calcd. (found) for $\mathrm{C}_{15} \mathrm{H}_{8} \mathrm{ClN}_{3} \mathrm{O}_{4}$ : C, 54.65 (54.70); $\mathrm{H}, 2.45$ (2.40); N, 12.75 (12.70); Cl, 10.75 (10.66).

\section{7-Chloro-6-(phenylamino)quinoline-5,8-dione 6b}

Procedure A. 6,7-Dichloro-5,8-quinolinequinone 5a $(0.88$ mmol, $200 \mathrm{mg}$ ), aniline $(0.86 \mathrm{mmol}, 80 \mathrm{mg}), \mathrm{NaOt}-\mathrm{Bu}(1.06$ mmol, $101 \mathrm{mg}, 1.2 \mathrm{eq}), 170 \mathrm{~min}$. The product (dark red solid) m.p. $314-316{ }^{\circ} \mathrm{C}$ was obtained in $173 \mathrm{mg}(0.608 \mathrm{mmol})$; yield: $85 \%$.

The ultraviolet maximum absorption bands in methanol: $\mathrm{nm}$ (logE), 600 (0.039), 620 (0.015), 630 (0.013), 640 (0.010); ${ }^{1} \mathrm{H}$ NMR $\left(400 \mathrm{MHz}, \mathrm{CDCl}_{3}\right): \delta=8.8(\mathrm{~d}, J=4 \mathrm{~Hz}, 1 \mathrm{H}), 8.4$ 
(d, $J=6.8 \mathrm{~Hz}, 1 \mathrm{H}), 7.7(\mathrm{dd}, J=4.8, J=7.6 \mathrm{~Hz}, 1 \mathrm{H}), 7.2(\mathrm{dd}$, 2H), $7.1(\mathrm{~m}, 3 \mathrm{H}) \mathrm{ppm} ;{ }^{13} \mathrm{C} \mathrm{NMR}\left(100 \mathrm{MHz}, \mathrm{CDCl}_{3}\right): \delta=161.51$ $(\mathrm{C}=\mathrm{O}(\mathrm{C}-5)), 153.89$ (C-1), 151.33 (C-7), 1136.31 (C-9), 136.12 (C-10), 133.82 (C-3), 128.02 (C-14 \& C-12), 125.58 (C-11 \& C-15), 113.41 (C-13), 112.36 (C-6) ppm; IR 1558 $(\mathrm{C}=0), 1512(\mathrm{NH}) \mathrm{cm}^{-1}$; MS 284.04, 285.04; Anal. Calcd. (found) for $\mathrm{C}_{15} \mathrm{H}_{9} \mathrm{ClN}_{2} \mathrm{O}_{2}$ : C, 63.28 (63.33); $\mathrm{H}, 3.19$ (3.20); N, 9.84 (9.93); Cl, 12.45 (12.70).

\section{7-Chloro-6-(o-tolylamino)quinoline-5,8-dione 6c}

Procedure B. 6,7-Dichloro-5,8-quinolinequinone 5a (0.44 mmol, $100 \mathrm{mg}$ ), $o$-toluidine (0.53 mmol, $57 \mathrm{mg}$ ), $\mathrm{K}_{2} \mathrm{CO}_{3}(0.62 \mathrm{mmol}$, $86 \mathrm{mg}, 1.4 \mathrm{eq}), 70 \mathrm{~min}$. The product (dark red solid) m.p. $316-318{ }^{\circ} \mathrm{C}$ was obtained in $112 \mathrm{mg}$ (0.373 mmol); yield: $85 \%$.

The ultraviolet maximum absorption bands in methanol: $\mathrm{nm}$ (logE), 600 (1.099), 620 (0.819), 630 (0.727), 640 (0.654); IR $1678(\mathrm{C}=0), 1504(\mathrm{~N}-\mathrm{H}), 1307\left(\mathrm{CH}_{3}\right), 3250 \mathrm{br} \mathrm{cm}{ }^{-1}$; MS 298.05, 263.08; Anal. Calcd. (found) for $\mathrm{C}_{16} \mathrm{H}_{11} \mathrm{ClN}_{2} \mathrm{O}_{2}$ : C, 64.33 (64.30); H, 3.71 (3.64); N, 9.38 (9.32); Cl, 11.87 (11.68).

7-Bromo-6-[(4-nitrophenyl)amino]quinoline-5,8-dione 6d Procedure A. 6,7-Dibromo-5,8-quinolinequinone 5b $(0.63$ mmol, $200 \mathrm{mg}$ ), 4-nitroaniline (2.52 mmol, $348 \mathrm{mg}$ ), $\mathrm{NaOt}$-Bu (0.76 mmol, $73 \mathrm{mg}, 1.2 \mathrm{eq}), 75 \mathrm{~min}$. The product (red solid) m.p. $238-240{ }^{\circ} \mathrm{C}$ was obtained in $203 \mathrm{mg}$ (0.54 mmol), yield: $86 \%$.

The ultraviolet maximum absorption bands in methanol: $\mathrm{nm}$ (logE), 600 (2.697), 620 (2.7), 630 (2.282) 640 (2.389); ${ }^{1} \mathrm{H}$ $\operatorname{NMR}\left(400 \mathrm{MHz}, \mathrm{CDCl}_{3}\right.$ ): $\delta=9.0(\mathrm{dd}, J=1.6, J=4.8 \mathrm{~Hz}, 1 \mathrm{H})$, $8.4(\mathrm{dd}, J=1.6, J=7.6 \mathrm{~Hz}, 1 \mathrm{H}), 8.2(\mathrm{~m}, 2 \mathrm{H}), 7.6(\mathrm{dd}, J=4.8$, $J=8.0 \mathrm{~Hz}, 2 \mathrm{H}), 7.1(\mathrm{~m}, 2 \mathrm{H}) \mathrm{ppm} ;{ }^{13} \mathrm{C} \mathrm{NMR}\left(100 \mathrm{MHz}, \mathrm{CDCl}_{3}\right)$ : $\delta=206.4(\mathrm{C}=\mathrm{O}(\mathrm{C}-7)), 207.0(\mathrm{C}=\mathrm{O}(\mathrm{C}-4)), 161.2(\mathrm{C}-6), 153.9$ (C-1), 149.2 (C-8), 114.6 (C-9 \& C-13), 134.0 (C-10 \& C-12), 136.3 (C-11), 136.2 (C-3), 128.0 (C-15), 127.6 (C-14), 112.4 (C-5) ppm; IR 1712 (C = 0), $1419\left(\mathrm{NO}_{2}\right) \mathrm{cm}^{-1}$; MS 371.94, 328.00, $239.04\left(\mathrm{M}^{+}\right)$; Anal. Calcd. (found) for $\mathrm{C}_{15} \mathrm{H}_{8} \mathrm{BrN}_{3} \mathrm{O}_{4}$ : C, 48.15 (48.18); H, 2.16 (2.15); N, 11.23 (11.20); Br, 21.36 (21.30).

\section{7-Bromo-6-[phenylamino]quinoline-5,8-dione $6 \mathrm{e}$}

Procedure A. 6,7-Dibromo-5,8-quinolinequinone $\mathbf{5 b}(0.63 \mathrm{mmol}$, $200 \mathrm{mg}$ ), aniline (0.76 mmol, $71 \mathrm{mg}), \mathrm{NaOt}-\mathrm{Bu}(0.76 \mathrm{mmol}$, $73 \mathrm{mg}, 1.2 \mathrm{eq}$ ), $60 \mathrm{~min}$. The product (dark red solid) m.p. 254$256{ }^{\circ} \mathrm{C}$ was obtained in $187 \mathrm{mg}$ ( $\left.0.568 \mathrm{mmol}\right)$; yield: $90 \%$. The ultraviolet maximum absorption bands in methanol: $\mathrm{nm}$ (logE), 600 (0.045), 620 (0.019), 630 (0.010) 640 (0.009); ${ }^{1} \mathrm{H}$ NMR (400 MHz, $\left.\mathrm{CDCl}_{3}\right): \delta=8.8(\mathrm{dd}, J=2, J=4.8 \mathrm{~Hz}, 1 \mathrm{H})$, $8.4(\mathrm{dd}, J=1.6, J=8 \mathrm{~Hz}, 1 \mathrm{H}), 7.7(\mathrm{dd}, J=4.8, J=8 \mathrm{~Hz}, 1 \mathrm{H})$ 7.3 (dd, 2H), 7.1 (m, 3H) ppm; IR 1550 (C = 0), $1516(\mathrm{NH})$; $2600 \mathrm{br} \mathrm{cm}^{-1}$; MS 329.0, $331.0\left(\mathrm{M}^{+}\right)$; Anal. Calcd. (found) for $\mathrm{C}_{15} \mathrm{H}_{9} \mathrm{BrN}_{2} \mathrm{O}_{2}$ : C, 54.74 (54.80); H, 2.76 (2.81); N, 8.51 (8.57); $\mathrm{Br}, 24.28$ (24.22).

7-Bromo-6-(o-tolylamino)quinoline-5,8-dione $6 f$

Procedure B. 6,7-Dibromo-5,8-quinolinequinone 5b $\quad 0.32$ mmol, $100 \mathrm{mg}$ ), $o$-toluidine $(0.38 \mathrm{mmol}, 41 \mathrm{mg}), \mathrm{K}_{2} \mathrm{CO}_{3}(0.62$ mmol, $86 \mathrm{mg}, 1.4 \mathrm{eq}$ ), $90 \mathrm{~min}$. The product (red solid) m.p. $216-218{ }^{\circ} \mathrm{C}$ was obtained in $98 \mathrm{mg}$ (0.284 mmol); yield: $90 \%$. The ultraviolet maximum absorption bands in methanol: $\mathrm{nm}$ (logE), 600 (0.393), 620 (0.272), 630 (0.249), 640 (0.233); IR 1739 (C = 0), $1373 \mathrm{~cm}^{-1}$; MS 143.14, 239.12, 313.15, $341.06\left(\mathrm{M}^{-}\right)$; Anal. Calcd. (found) for $\mathrm{C}_{16} \mathrm{H}_{11} \mathrm{BrN}_{2} \mathrm{O}_{2}$ : C, 56.00 (56.30); H, 3.23 (3.19); N, 8.16 (8.21); Br, 23.28 (23.17). 6,7-Bis[(4-chloro-2-nitrophenyl)amino]quinoline-5,8dione $\mathbf{6 g}$

Procedure A. 6,7-Dibromo-5,8-quinolinequinone 5b (0.63 mmol, $200 \mathrm{mg}$ ), 4-chloro-2-nitroaniline (2.52 mmol, $435 \mathrm{mg}$ ), $\mathrm{NaOt}$-Bu (0.76 mmol, $73 \mathrm{mg}, 1.2 \mathrm{eq}), 190 \mathrm{~min}$. The product (red solid) m.p. $286-288{ }^{\circ} \mathrm{C}$ was obtained in $268 \mathrm{mg}(0.536$ mmol); yield: $85 \%$.

The ultraviolet maximum absorption bands in methanol: $\mathrm{nm}$ (logE), 600 (0.043), 620 (0.017), 630 (0.008) 640 (0.007); ${ }^{1} \mathrm{H}$ NMR (400 MHz, MeOH): $\delta=8.92(\mathrm{dd}, J=1.6, J=4.8 \mathrm{~Hz}$, $1 \mathrm{H}), 8.91(\mathrm{~s}, 2 \mathrm{H}), 8.36$ (ddd, $J=1.6, J=7.6 \mathrm{~Hz}, 1 \mathrm{H}), 8.32(\mathrm{~m}$, 2H), $7.60(\mathrm{dd}, J=4.8, J=8 \mathrm{~Hz}, 1 \mathrm{H}), 7.58(\mathrm{~s}, 2 \mathrm{H}), 7.56(\mathrm{~m}, 2 \mathrm{H})$ ppm; IR 1700 ( $\mathrm{C}=0), 1500\left(\mathrm{NO}_{2}\right), 2600 \mathrm{~cm}^{-1}$; MS 130.99; $501.97\left(\mathrm{M}^{+}\right)$; Anal. Calcd. (found) for $\mathrm{C}_{21} \mathrm{H}_{11} \mathrm{Cl}_{2} \mathrm{~N}_{5} \mathrm{O}_{6}$ : C, 50.42 (50.71); H, 2.22 (2.14); N, 14.00 (14.03); Cl, 14.17 (14.23).

\section{2-Chloro-3-((4-nitrophenyl)amino)naphthalene-1,4-}

\section{dione 8a}

Procedure B. 2,3-Dichloro-1,4-naphthoquinone $(0.88 \mathrm{mmol}$, $200 \mathrm{mg}$ ), 4-nitroaniline (1.10 mmol, $152 \mathrm{mg}), \mathrm{K}_{2} \mathrm{CO}_{3}(1.23$ mmol, $170 \mathrm{mg}, 1.4 \mathrm{eq}), 130 \mathrm{~min}$. The product (dark red solid) m.p. $318-320{ }^{\circ} \mathrm{C}$ was obtained in $243 \mathrm{mg}(0.74 \mathrm{mmol})$; yield: $84 \%$.

The ultraviolet maximum absorption bands in methanol: $\mathrm{nm}$ (logE), 600 (0.082), 620 (0.039), 630 (0.035), $640(0.018) ;{ }^{1} \mathrm{H}$ NMR (400 MHz, $\mathrm{CDCl}_{3}$ ): $\delta=8.2(\mathrm{dd}, 2 \mathrm{H}), 7.28(\mathrm{dd}, 2 \mathrm{H}), 7.23$ (dd, 2H), 7.2 (s, 1H), 6.7 (d, 2H) ppm; IR 1712 (C = 0), 1419 $\left(\mathrm{NO}_{2}\right), 663$ ( $\mathrm{C}=\mathrm{C}$ in ring) $\mathrm{cm}^{-1}$; MS 264.98, 298.03, 313.98, 328.02; Anal. Calcd. (found) for $\mathrm{C}_{16} \mathrm{H}_{9} \mathrm{ClN}_{2} \mathrm{O}_{4}$ : C, 58.46 (58.41); H, 2.76 (2.82); N, 8.52 (8.61); Cl, 10.78 (10.93).

\section{2-Chloro-3-(phenylamino)naphthalene-1,4-dione 8b}

Procedure A. 2,3-Dichloro-1,4-naphthoquinone (0.88 mmol, 200 $\mathrm{mg}$ ), aniline (1.10 mmol, $102 \mathrm{mg}$ ), $\mathrm{NaOt}$-Bu (1.06 mmol, 101 $\mathrm{mg}, 1.2 \mathrm{eq}$ ), $120 \mathrm{~min}$. The product (dark red solid) m.p. 314$316{ }^{\circ} \mathrm{C}$ was obtained in $225 \mathrm{mg}$ (0.793 mmol); yield: $90 \%$.

The ultraviolet maximum absorption bands in methanol: $\mathrm{nm}$ (logE), 600 (0.366), 620 (0.276), 630 (0.252), 640 (0.214); ${ }^{1} \mathrm{H}$ NMR (400 MHz, $\left.\mathrm{CDCl}_{3}\right): \delta=8.1(\mathrm{dd}, 2 \mathrm{H}), 8.1(\mathrm{dd}, 2 \mathrm{H})$, 7.7 (dd, 2H), 7.6 (dd, 4H) ppm; IR 1739 (C = 0), 2340 br cm ${ }^{-1}$; MS 144.04, 248.07, 283.04, 285.04, $\mathrm{M}^{+}$; Anal. Calcd. (found) for $\mathrm{C}_{16} \mathrm{H}_{10} \mathrm{ClNO}_{2}$ : C, 67.74 (67.70); $\mathrm{H}, 3.55$ (3.51); N, 4.94 (4.89); Cl, 12.50 (12.37).

\section{2-Chloro-3-(o-tolylamino)naphthalene-1,4-dione 8c}

Procedure B. 2,3-Dichloro-1,4-naphthoquinone $(0.44 \mathrm{mmol}$, $100 \mathrm{mg}$ ), $o$-toluidine $(0.53 \mathrm{mmol}, 57 \mathrm{mg}), \mathrm{K}_{2} \mathrm{CO}_{3}(0.61 \mathrm{mmol}$, $85 \mathrm{mg}, 1.4 \mathrm{eq}$ ), $130 \mathrm{~min}$. The product (dark red solid) m.p. $316-318{ }^{\circ} \mathrm{C}$ was obtained in $105 \mathrm{mg}$ (0.352 mmol); yield: $80 \%$. 
The ultraviolet maximum absorption bands in methanol: nm (logE), 600 (0.031), 620 (0.022), 630 (0.024), 640 (0.017); IR $1674(\mathrm{C}=0), 1508(\mathrm{NH}), 1477 \mathrm{~cm}^{-1}$; MS 121.02, 161.05, 191.02, $297.93 \mathrm{M}^{+}$; Anal. Calcd. (found) for $\mathrm{C}_{17} \mathrm{H}_{12} \mathrm{ClNO}_{2}$ : C, 68.58 (68.70); H, 4.06 (4.00); N, 4.70 (4.71); $\mathrm{Cl}_{\text {, }}$ 11.91 (11.98).

\section{REFERENCES}

[1] Keyari CM, Kearns AK, Duncan NS, Eickholt EA, Abbott G, Beall HD, Diaz P. Synthesis of new quinolinequinone derivatives and preliminary exploration of their cytotoxic properties. J Med Chem. 2013;56:3806-19. doi:10.1021/jm301689x

[2] Porter TH, Skelton FS, Folkers K. Synthesis of new alkylaminoand alkylaminomethyl-5,8-quinolinequinones as inhibitors of coenzyme Q and as antimalarials. J Med Chem. 1972;15:34-6.

[3] Fryatt T, Pettersson HI, Gardipee WT, Bray KC, Green SJ, Slawin AM, Beall HD, Moody CJ. Novel quinolinequinone antitumor agents: structure-metabolism studies with $\mathrm{NAD}(\mathrm{P}) \mathrm{H}:$ quinone oxidoreductase (NQ01). Bioorg Med Chem. 2004;12:1667-87. doi:10.1016/j.bmc.2004.01.021

[4] Donohoe TJ, Jones CR, Barbosa LCA. Total synthesis of ( \pm )-streptonigrin: De Novo construction of a Pentasubstituted pyridine using ring-closing metathesis. J Am Chem Soc. 2011;133:1641821. doi:10.1021/ja207835w

[5] Boger DL, Cassidy KC, Nakahara S. Total synthesis of streptonigrone. J Am Chem Soc. 1993;115:10733-41. doi:10.1021/ja000 $76 \mathrm{a} 035$

[6] Nissen F, Detert H. Total Synthesis of Lavendamycin by a [2+2 +2] Cycloaddition. Eur J Org Chem. 2011;2011:2845-53. doi:10. 1002/ejoc.201100131

[7] Mulchin BJ, Newton CG, Baty JW, Grasso CH, Martin WJ, Walton MC, Dangerfield EM, Plunkett $\mathrm{CH}$, Berridge MV, Harper JL, Timmer MS, Stocker BL. The anti-cancer, anti-inflammatory and tuberculostatic activities of a series of 6,7-substituted5,8-quinolinequinones. Bioorg Med Chem. 2010;18:3238-51. doi:10.1016/j.bmc.2010.03.021

[8] Ryu C-K, Kim H-J. A series of 6-(N-arylamino)-7-chloro-5,8-quinolinedione derivatives was newly synthesized for the evaluation of antifungal activities. Arch Pharm Res. 1994;17:139-44.

[9] Ryu CK. U.S. Patent No. 6,818,653, 2004.

[10] Wang Z. Comprehensive organic name reactions and reagents. Wiley; 2010. 575-81 pp. doi:10.1002/9780470638859

[11] Vorogushin AV, Huang X, Buchwald SL. Use of tunable ligands allows for intermolecular Pd-catalyzed $\mathrm{C}-\mathrm{O}$ bond formation. J Am Chem Soc. 2005;127:8146-9. doi:10.1021/ja050471r
[12] Paul F, Patt J, Hartwig JF. Palladium-catalyzed formation of carbon-nitrogen bonds. Reaction intermediates and catalyst improvements in the hetero cross-coupling of aryl halides and tin amides. J Am Chem Soc. 1994;116:5969-70. doi:10.1021/ ja00092a058

[13] Huang X, Anderson KW, Zim D, Jiang L, Klapars A, Buchwald SL. Expanding Pd-catalyzed $\mathrm{C}-\mathrm{N}$ bond-forming processes: the first amidation of aryl sulfonates, aqueous amination, and complementarity with $\mathrm{Cu}$-catalyzed reactions. J Am Chem Soc. 2003; 125:6653-5. doi:10.1021/ja035483w

[14] Galardon E, Ramdeehul S, Brown JM, Cowley A, Hii KK, Jutand A. Profound steric control of reactivity in aryl halide addition to Bisphosphane palladium(0) complexes. Angew Chem Int Ed. 2002;41:1760-3. doi:10.1002/1521-3773(20020517)41:10<17 60::AID-ANIE1760>3.0.CO;2-3

[15] Fors BP, Krattiger P, Strieter E, Buchwald SL. Water-mediated catalyst Preactivation: an efficient protocol for $\mathrm{C}-\mathrm{N}$ crosscoupling reactions. Org Lett. 2008;10:3505-8. doi:10.1021/ol80 1285g

[16] Pratt YT. Quinolinequinones. VI. Reactions with aromatic amines. J Org Chem. 1962;27:3905-10. doi:10.1021/jo0105 $8 \mathrm{a} 036$

\section{COMPETING INTERESTS}

The authors declare no competing interests.

\section{PUBLISHING NOTES}

(C) 2014 S.A. Egu et al. This work has been published open access under Creative Commons Attribution License CC BY 4.0, which permits unrestricted use, distribution, and reproduction in any medium, provided the original work is properly cited. Conditions, terms of use and publishing policy can be found at www.scienceopen.com.

Please note that this article may not have been peer reviewed yet and is under continuous post-publication peer review. For the current reviewing status please click here or scan the QR code on the right.

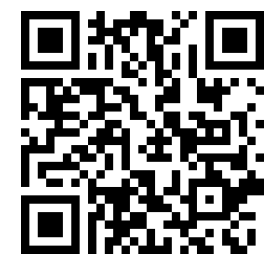

\section{scienceOPEN.com}

